\title{
The association of carotid intima-media thickness with body mass index and cortisol level in fibromyalgia syndrome
}

\author{
Tuba Tülay Koca ${ }^{1}$, Ayla Çimen ${ }^{2} \odot$ \\ ${ }^{1}$ Department of Physical Medicine and Rehabilitation, Sütçü Imam University School of Medicine, Kahramanmaraş, Turkey \\ ${ }^{2}$ Department of Radiology, Malatya Training and Research Hospital, Malatya, Turkey
}

DOI: $10.18621 /$ eurj.376343

\begin{abstract}
Objective: Patients with fibromyalgia syndrome (FMS) may have the risk for atherosclerotic disease because of chronic stress, disrupted neuroendocrine axis, high BMI and inflammation. This is study aimed to research the stress and the stress-related diseases in patients with FMS by measuring obesity, morning serum cortisol level, and carotid intima-media thickness (CIMT), which is the early indicator of the atherosclerosis.

Methods: This is a case-control study. It is included 57 patients who were diagnosed with FMS according to 2010 American College of Rheumatology (ACR) Criteria and visited the outpatient clinic in February 2016. As a control group, 36 healthy females who visited the outpatient clinic in the same month were included. Carotid intima-media thickness and cortisol levels were analysed.

Results: The present study included 93 females (between 15 and 79 years old) in total. The mean age of the 57 females with FMS and 37 healthy females was $44.5 \pm 10.6$ years and $47.9 \pm 18.5$ years, respectively $(p=$ 0.260). The mean CIMT value of the FMS and control groups was $0.64 \pm 0.17 \mathrm{~mm}$ and $0.59 \pm 0.16 \mathrm{~mm}$, respectively $(p=0.170)$. The morning blood cortisol level in the FMS and control groups was $7.8 \pm 3.3 \mathrm{ng} / \mathrm{dL}$ and $8.7 \pm 2.8 \mathrm{ng} / \mathrm{dL}$, respectively ( $p=0.460$ ). The CIMT and morning blood cortisol level were not correlated in the FMS group.

Conclusion: According to the present study, CIMT value and morning blood cortisol level were not found significantly different in FMS group, and also were not found correlated with each other.
\end{abstract}

Keywords: Atherosclerosis, carotid intima-media thickness, fibromyalgia syndrome

Received: January 8, 2018; Accepted: March 11, 2018; Published Online: April 11, 2018

I ntima-media thickness (IMT) is also called as intimal medial thickness. It is the measure of the thickness of the innermost two layers of the arterial wall called tunica intima and tunica media. This measurement is usually done by external ultrasonography (US) and rarely by the internal invasive US catheters [1].

The role of the IMT is not clear, and it is rarely used in clinic. Carotid IMT (CIMT) is measured in various epidemiologic and clinical studies. A significant correlation was found between CIMT and several diseases such as type 2 diabetes mellitus, disrupted glucose tolerance, familial hypercholesterolemia, rheumatoid arthritis, and nonalcoholic fatty liver disease, as well as air pollution. An IMT value higher than $0.9-1 \mathrm{~mm}$ is an indicator of atherosclerosis and other cardiovascular diseases [2, 3]. 
Fibromyalgia syndrome (FMS) is characterized by chronic skeletal and muscular pain, and its pathogenesis still is not known. In clinical studies, endocrinologic responses differ in patients with FMS, but clinical findings are conclusive, too. The dysfunction of hypothalamus-pituitary-adrenal axis is commonly seen [4]. Commonly, abnormal cortisol levels and corticotropin releasing hormone response are reported in FMS patients [5, 6].

Obesity and overweight, often present in FMS patients, are related to the severity of FMS worsening the quality of life in terms of higher pain, fatigue, worsened sleep quality and higher incidence of mood disorders. Weight control is thus an effective tool to improve the symptoms [7]. To best of our knowledge, there are no other publications in the literature that analysis atherosclerosis and CIMT in FMS patients. This study explored the association of CIMT with body mass index (BMI) and cortisol level in fibromyalgia syndrome.

\section{METHODS}

This study included 57 patients who were diagnosed with FMS according to 2010 American College of Rheumatology Criteria (ACR) and visited the Physical Medicine and Rehabilitation outpatient clinic on February 2016 [8]. Only patients attended at between 7:00 am and 9:00 am, included the study. Any patient who has any disease related hypothalamuspituitary-adrenal axis disfunction, serious atheroscleosis cardiac disease, using hormone supplement was excluded from the study.

As a control group, 36 healthy females who visited the outpatient clinic in the same month were included. The cortisol level of the patients was determined from their brachial venous blood samples. The basal cortisol level was taken as 6.2-19.4 $\mathrm{ng} / \mathrm{dL}$ (7:00-9:00 am). Data on patient age, height, weight, BMI, and medical history (hypertension and diabetes) were recorded.

The thickness of the bilateral common carotid artery was measured by the same radiologist (CIMT assessor was blinded) using both ultrasound (US) and a high-resolution, real-time scanner with $7.5 \mathrm{MHz}$ transducer. The measurements were taken when the patients were in the supine position, and maximum IMT values were recorded. The patients whose IMT value was higher than $1 \mathrm{~mm}$ formed the risk group.

\section{Statistical Analysis}

SPSS 17 (SPSS Statistics for Windows, Version 17.0. Chicago: SPSS Inc, USA) was used for statistical analysis. The normal distribution of the data was evaluated using Kolmogorov-Smirnov test. Some descriptive tests were used for the descriptive data. For each parameter, a comparison was done between the two groups. The t test was used for the groups that showed normal distribution. The Mann-Whitney U test was used for the groups that did not show normal distribution. A $p$ value $<0.05$ was considered statistically significant. The study has been done in accordance with the principles of Helsinki declaration.

\section{RESULTS}

This study included 93 females (between 15 and 79 years old) in total. The mean age of the 57 females with FMS and 37 healthy females was $44.5 \pm 10.6$ years and $47.9 \pm 18.5$ years, respectively $(p=0.260)$. The mean BMI value of the FMS and control groups was $28.6 \pm 4.7 \mathrm{~kg} / \mathrm{m} 2$ and $28.1 \pm 5.4 \mathrm{~kg} / \mathrm{m} 2,(p=$ $0.490)$ respectively. The descriptive characteristics for the groups are shown in Table 1. Diabetes mellitus was detected in five $(5.3 \%)$ patients (two FMS/three control), and hypertension was detected in six (6.4\%) patients (four FMS/two control).

The mean morning blood cortisol level in the FMS and control groups was $7.8 \pm 3.3 \mathrm{ng} / \mathrm{dL}$ and $8.7 \pm 2.8$ $\mathrm{ng} / \mathrm{dL}$, respectively. No statistical difference was found in the morning blood cortisol level of these two groups ( $p=0.460)$ (Figure 1). The mean CIMT value of the FMS and control groups was $0.64 \pm 0.17 \mathrm{~mm}$

Table 1. The descriptive data of the patients according to the groups

\begin{tabular}{lccc}
\hline & $\begin{array}{c}\text { Control } \\
(\mathbf{n}=\mathbf{3 6})\end{array}$ & $\begin{array}{c}\text { FMS } \\
(\mathbf{n}=\mathbf{5 7})\end{array}$ & $\boldsymbol{p}$ value \\
Age & $47.9 \pm 18.5$ & $44.5 \pm 10.6$ & 0.260 \\
BMI & $28.15 \pm 5.4$ & $28.69 \pm 4.78$ & 0.490 \\
Cortisol & $8.71 \pm 2.81$ & $7.85 \pm 3.34$ & 0.460 \\
CIMT & $0.59 \pm 0.16$ & $0.64 \pm 0.17$ & 0.170 \\
\hline
\end{tabular}

Data are shown as mean \pm standard deviation. BMI $=$ body mass index, CIMT = carotid intima-media thickness, FMS = fibromyalgia syndrome 


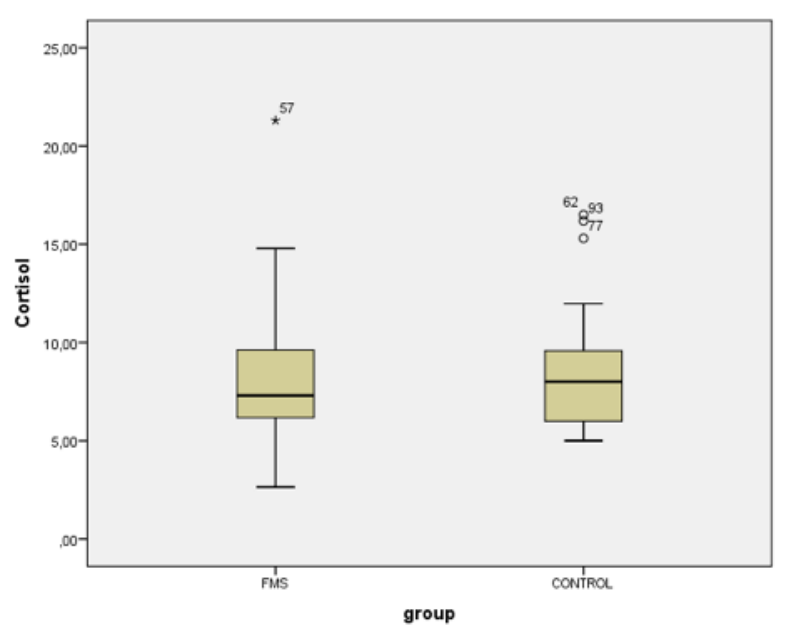

Figure 1. The distribution of serum cortisol in fibromyalgia syndrome and control group.

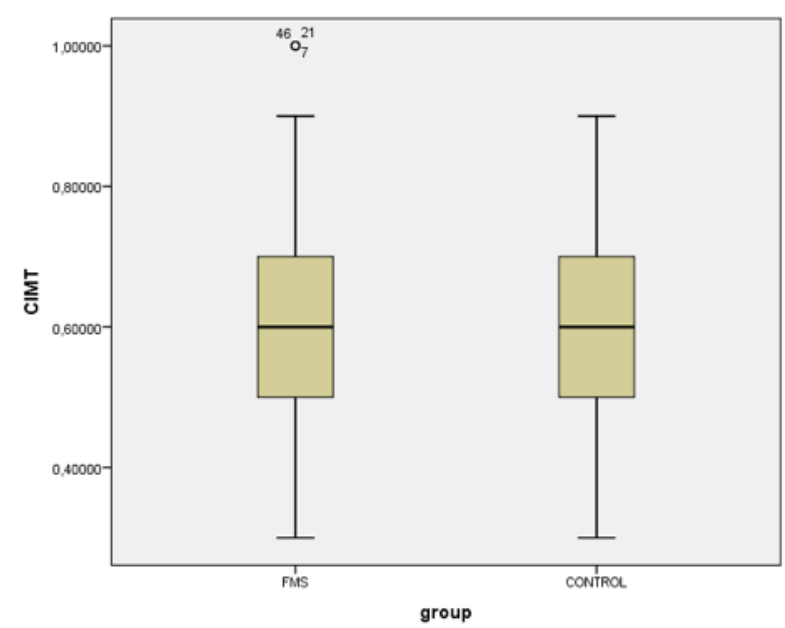

Figure 2. The distribution of carotid intima media thickness (CIMT) in fibromyalgia syndrome and control group.

and $0.59 \pm 0.16 \mathrm{~mm}$, respectively, with no statistically significant difference $(p=0.170)$ (Figure 2). The CIMT and morning blood cortisol level were not correlated in FMS group $(p=0.500)$. The CIMT values were positively correlated with age $(p<0.01)$ and BMI in all group $(p<0.01)$. In the FMS group, 3 $(5.5 \%)$ subjects; in the control group 0 subjects had CIMT $>1.00 \mathrm{~mm}(p=0.083)$.

\section{DISCUSSION}

According to the study, we did not find any significant difference between the results of morning cortisol, CIMT and BMI in the FMS group with control. CIMT was positively correlated with age and BMI.

IMT is usually used for detecting atherosclerotic diseases. It was first described by Pignoli [9] in 1984. IMT became a useful tool to monitor the noninvasive changes on the artery wall [9]. IMT is a complex process and depends on several factors such as local hemodynamics, blood pressure, and stres [1-4].

The symptoms of chronic stress-related diseases, such as obesity, hyperactivity in $\mathrm{CRH}$ neurons, disrupted cortisol secretion on a daily basis, common anxiety disruption, obsessive-compulsive personality and Atype personality disorder, sleep disorder, and chronic pain, are significant in the patients with FMS [10-12]. Chronic pain and stress-related deviations in hormonal secretion occur in patients with FMS. Hypothalamiccorticotrophin-releasing hormone $(\mathrm{CRH})$ neurons are activated in the central nervous system depending on chronic stress [10-12].

Since the sleep pattern is disrupted in women with FMS, it may lead to some changes in the circadian rhythm and disrupt the serum cortisol secretion. The circadian oscillation of the cortisol is abnormal in women with FMS [10-12]. A study by Fatima et al. [11] found the cortisol level in women with FMS to be significantly higher at night. In both genders, testosterone and DHEAS were found negatively correlated with cortisol [11]. To be able to detect hormonal changes in patients with FMS, Riedel et al. [10] injected CRH into their patients and observed high cortisol, low insuline like growth factor (IGF)-1 and triiodothyronine (T3), increase in the follicule stimulating hormon (FSH) level, and decrease in the basal estrogen level. Yeung et al. [12] also observed a more linear cortisol profile in adult patients with FMS who were exposed to childhood neglect. Riva et al. [4] measured the saliva cortisol level in patients with FMS who had pain, stress, and sleep disorders with depression. Despite these severe complaints, they found their saliva cortisol levels low, especially in the morning and the rest of the day [4]. In the present study, no significant difference was found in the morning cortisol level (measured at 7:00-9:00 am) between the two groups. Also we didnt find any relation with CIMT and BMI.

Since most of the women with FMS are overweight, BMI-related hormonal changes may be seen in these patients [13]. We observed no significant 
difference in the BMI values of the two groups in the present study. This may be because of being overweight, which is common in Turkish female population. Additionally, a positive correlation was noted between BMI and CIMT values. In the study by Rus et al. [13]cardiovascular disease-related biomarkers such as C-reactive protein and apolipoprotein B levels were found to be higher in women with FMS compared with the control group. Since the CIMT level is usually higher in obese individuals, CIMT can play an important role in detecting future pre-atherosclerotic changes [14]. Detecting atherosclerotic symptoms can take years, but it is important to identify them early especially in individuals who carry a high atherosclerotic risk [15]. FMS is used to be classified in non-enflammatory soft tissue diseases, but stress and stress-related diseases may induce an chronic inflammation causative for atherosclerosis. We found no significant difference in CIMT and cortisol levels in FMS group. But CIMT was positively correlated with age and BMI. In a similar study, Eller et al. [16] measured CIMT levels of 95 participants and detected a significantly negative correlation between CIMT and both age and high HDL in males and also a correlation between CIMT and morning cortisol level. We observed that the association between psychosocial stress and CIMT value was investigated in a few researches. Few number of studies reporting a correlation between morning cortisol level and IMT are available. Eller et al. [15] found a correlation between IMT and cortisol level in the saliva of women and indicated that a high morning cortisol might be correlated with a low CIMT.

Atherosclerosis is an important risk factor for stroke development. Approximately $25 \%$ to $40 \%$ of patients with stroke, largely younger patients, are not associated with any conventional stroke risk factors. Neuropsychiatric diseases like FMS might enhance stroke development, possibly through inflammation and atherosclerosis. In the study by Tseng et al. [17] the outcomes imply that FMS is a significant risk factor for stroke and that patients with FMS, particularly younger patients, require close attention and rigorous measures for preventing stroke. Obesity causes various complications associated with high inflammation [18]. FMS affects the quality of life of these young and productive women negatively as a reason of mainly obesity, hormonal disfunction, sleep and mood disorders etc.[19].

\section{Limitations}

The limitations of this study include the small sample size, single-site location of the investigation. All these factors may limit the generalizability of the results. Additionally, if the parameters of inflammation were taken into consideration, better results about atherosclerosis in FMS could be obtained.

\section{CONCLUSION}

Patients with FMS may harbor the risk for atherosclerotic disease cause of chronic stress, neuroendocrine disruption, high BMI and inflammation. As an easily accessible and noninvasive method, CIMT and the morning cortisol secretion due to stress and changes in hormonal levels might be useful in the early diagnosis of atherosclerosis.

According to the present study, CIMT value and morning blood cortisol level were not found significantly different in FMS group, and also were not found correlated with each other.

With contradictory results about cortisol levels in FMS patients in the literature, to the best of our knowledge the present study was the first to research the association between CIMT with cortisol and BMI in FMS patients.

\section{Conflict of interest}

The authors disclosed no conflict of interest during the preparation or publication of this manuscript.

\section{Financing}

The authors disclosed that they did not receive any grant during conduction or writing of this study.

\section{REFERENCES}

[1] de Groot E, van Leuven SI, Duivenvoorden R, Meuwese MC, Akdim F, Bots ML, et al. Measurement of carotid intima-media thickness to assess progression and regression of atherosclerosis. Nat Clin Pract Cardiovasc Med 2008;5:280-8.

[2] Costanzo P, Perrone-Filardi P, Vassallo E, Paolillo S, Cesarano P, Brevetti G, et al. Does carotid intima-media thickness regression predict reduction of cardiovascular events? A meta-analysis of 41 randomized trials. J Am Coll Cardiol 2010;56:2006-20. 
[3] Sharma K, Blaha MJ, Blumenthal RS, Musunuru K. Clinical and research applications of carotid intima-media thickness. Am J Cardiol 2009;103:1316-20.

[4] Riva R, Mork PJ, Westgaard RH, Rø M, Lundberg U. Fibromyalgia syndrome is associated with hypocortisolism. Int $\mathrm{J}$ Behav Med 2010;17:223-33.

[5] Semiz EA, Hizmetli S, Semiz M, Karadăg A, Adalı M, Tuncay MS, et al. Serum cortisol and dehydroepiandrosterone-sulfate levels after balneotherapy and physical therapy in patients with fibromyalgia. Saudi Med J 2016;37:544-50.

[6] Rossi A, Di Lollo AC, Guzzo MP, Giacomelli C, Atzeni F, Bazzichi L, et al. Fibromyalgia and nutrition: what news? Clin Exp Rheumatol 2015;33(1 Suppl 88):S117-25.

[7] Koca TT, Tanrikut E, Acet G, Talu B. Evaluation of sleep disorder and its effect on sexual dysfunction in patients with fibromyalgia syndrome. Turk J Obstet Gynecol 2016;13:167-71.

[8] Sarzi-Puttini P, Atzeni F, Masala IF, Salaffi F, Chapman J, Choy E. Are the ACR 2010 diagnostic criteria for fibromyalgia better than the 1990 criteria? Autoimmun Rev 2018;17:33-35.

[9] Pignoli P. Ultrasound B-mode imaging for arterial wall thickness measurement. Atherosclerosis Rev 1984;12:177-84.

[10] Riedel W, Layka H, Neeck G. Secretory pattern of GH, TSH, thyroid hormones, ACTH, cortisol, FSH, and LH in patients with fibromyalgia syndrome following systemic injection of the relevant hypothalamic-releasing hormones. Z Rheumatol 1998;57:81-7.

[11] Fatima G, Das SK, Mahdi AA, Verma NS, Khan FH, Tiwari AM, et al. Circadian rhythm of serum cortisol in female patients with fibromyalgia syndrome. Indian J Clin Biochem 2013;28:181-4.

[12] Yeung EW, Davis MC, Ciaramitaro MC. Cortisol profile mediates the relation between childhood neglect and pain and emotional symptoms among patients with fibromyalgia. Ann Behav Med 2016;50:87-97.

[13] Rus A, Molina F, Gassó M, Camacho MV, Peinado MÁ, Moral ML. Nitric oxide, inflammation, lipid profile, and cortisol in normal- and overweight women with fibromyalgia. Biol Res Nurs 2016;18:138-46. [14] Önal ZE, Soydan L, Öztürk HE, Sağ Ç, Gürbüz T, Nuhoğlu Ç, et al. Carotid intima media thickness in obese children: is there an association with hyperlipidemia? J Pediatr Endocrinol Metab 2016;29:157-62.

[15] Eller NH, Netterstrøm B, Hansen AM. Cortisol in urine and saliva: relations to the intima media thickness, IMT. Atherosclerosis 2001;159:175-85.

[16] Eller NH, Netterstrøm B, Allerup P. Progression in intima media thickness--the significance of hormonal biomarkers of chronic stress. Psychoneuroendocrinology 2005;30:715-23.

[17] Tseng CH, Chen JH, Wang YC, Lin MC, Kao CH. Increased risk of stroke in patients with fibromyalgia: a population-based cohort study. Medicine (Baltimore) 2016;95:e2860.

[18] Koca TT. Does obesity cause chronic inflammation? The association between complete blood parameters with body mass index and fasting glucose. Pak J Med Sci 2017;33:65-9.

[19] Tulay KT, Emrullah T, Aydin A, Ciledag OF. The effect of fibromyalgia syndrome to gravidity, parity and duration of breastfeeding; A prospective study from Turkey. Pak J Med Sci 2016;32:545-9. 